\title{
THE ZYGMUND MORSE-SARD THEOREM
}

\author{
Alec Norton
}

\begin{abstract}
The classical Morse-Sard Theorem says that the set of critical values of $f: \mathbf{R}^{n+k} \rightarrow \mathbf{R}^{n}$ has Lebesgue measure zero if $f \in C^{k+1}$. We show the $C^{k+1}$ smoothness requirement can be weakened to $C^{k+Z y g m u n d}$. This is corollary to the following theorem: For integers $n>m>r>0$, let $s=(n-r) /(m-r)$; if $f: \mathbf{R}^{n} \rightarrow$ $\mathbf{R}^{m}$ belongs to the Lipschitz class $\Lambda_{s}$ and $E$ is a set of rank $r$ for $f$, then $f(E)$ has measure zero.
\end{abstract}

\section{INTRODUCTION}

Let $f: \mathbf{R}^{n} \rightarrow \mathbf{R}^{m}$ be a differentiable function. Must the set of critical values of $f$ have measure zero? The answer is "yes" provided that $f$ is sufficiently smooth, and the classical theorem in this regard is the Morse-Sard theorem (often called simply "Sard's theorem" - A.P. Morse [6] proved the theorem in 1939 for the real-valued case; A. Sard [10] then extended that result to the vector-valued case.)

To state the theorem, we need some terminology. For $f$ as above, a point $x \in \mathbf{R}^{n}$ is called a critical point if the linear mapping $D f(x)$ is not surjective; a critical value is the image under $f$ of a critical point. The set of all critical values is a subset of the target space $\mathbf{R}^{m}$.

The Morse-Sard Theorem. [6,10]. Let $f: \mathbf{R}^{n} \rightarrow \mathbf{R}^{m}$ be of class $C^{k}$.

If $k \geq \max \{n-m+1,1\}$, then the set of critical values of $f$ has Lebesgue measure zero.

We henceforth restrict our attention to the case $n>m$; smoothness is not an issue when $n \leq m$. (In fact even measurability is not required if $n \leq m$. See Varberg [12].)

Prior to Morse's work, H. Whitney had established in a famous paper [13] that some differentiability requirement is necessary by constructing an example of a $C^{1}$ function $f: \mathbf{R}^{2} \rightarrow \mathbf{R}$ not constant on a connected set of critical points (and hence having a nontrivial interval of critical values). This example, and related ones in higher dimensions, shows that the smoothness hypothesis of the MorseSard theorem is sharp to the extent that one cannot replace the integer $n-m+1$ by any smaller integer. In fact, more is true: for every $n, m$ with $n>m>0$ there is a function $f \in \bigcap_{\alpha<1} \mathbf{C}^{n-m, \alpha}\left(\mathbf{R}^{n}, \mathbf{R}^{m}\right)$ whose critical value set contains an open set $[2,8]$.

(Notation: for $\alpha \in[0,1), k$ a nonnegative integer, we say $f \in C^{k, \alpha}\left(\mathbf{R}^{n}, \mathbf{R}^{m}\right)$ if $f: \mathbf{R}^{n} \rightarrow \mathbf{R}^{m}$ is of class $C^{k}$ and the $k$ th derivative $D^{k} f$ locally satisfies a Hölder condition with exponent $\alpha$. We say $f \in C^{k, 1}\left(\mathbf{R}^{n}, \mathbf{R}^{m}\right)$ if the $k$ th derivative is locally Lipschitz.) 
This paper addresses the question of whether the Morse-Sard theorem holds true for any natural smoothness class weaker than $C^{n-m+1}$. Because of the examples mentioned above, there is very little room for improvement. Nevertheless, S. Bates [1] has recently improved the statement to

The Lipschitz Morse-Sard Theorem (Bates). Let $n, m$ be positive integers with $n>m$. If $f \in C^{n-m, 1}\left(\mathbf{R}^{n}, \mathbf{R}^{m}\right)$ then the set of critical values of $f$ has Lebesgue measure zero.

Even weaker than the Lipschitz class is the so-called Zygmund class, defined as follows. We say that $f: \mathbf{R}^{n} \rightarrow \mathbf{R}^{m}$ is Zygmund if it is continuous and for every compact set $K \subset \mathbf{R}^{n}$, there is a constant $C$ such that if $x, x+h$, and $x-h$ belong to $K$, then

$$
|f(x+h)+f(x-h)-2 f(x)| \leq C|h| .
$$

We say that $f \in C^{k, Z}\left(\mathbf{R}^{n}, \mathbf{R}^{m}\right)$ if $f$ is $C^{k}$ and all of the $k$ th partial derivatives of $f$ are Zygmund.

For basic properties of the Zygmund class, see [5] or [11]. This class is very well known in harmonic analysis, and in fact there is a strong case (see [5]) that for many purposes in analysis the class $C^{k, Z}$ is more natural than the classical class $C^{k+1}$. This class also arises naturally in certain dynamical systems settings, e.g. $[9]$.

It will be slightly more convenient for us to use the "Lipschitz spaces" $\Lambda_{s}$ of harmonic analysis, defined as follows.

If $0<s<1$, then $f \in \Lambda_{s}\left(\mathbf{R}^{n}, \mathbf{R}^{m}\right)$ if $f: \mathbf{R}^{n} \rightarrow \mathbf{R}^{m}$ is continuous and

$$
\|f\|_{s} \equiv \sup _{x \in \mathbf{R}^{n}}|f(x)|+\sup _{x, h \in \mathbf{R}^{n}}|f(x+h)-f(x)| /|h|^{s}<\infty .
$$

We say $f \in \Lambda_{1}$ if $f$ is continuous and

$$
\|f\|_{1} \equiv \sup _{x \in \mathbf{R}^{n}}|f(x)|+\sup _{x, h \in \mathbf{R}^{n}}|f(x+h)+f(x-h)-2 f(x)| /|h|<\infty .
$$

Inductively, for $s>1$, we say $f \in \Lambda_{s}\left(\mathbf{R}^{n}, \mathbf{R}^{m}\right)$ if $f \in C^{k}\left(\mathbf{R}^{n}, \mathbf{R}^{m}\right)$, where $k$ is the largest integer less than $s$, and

$$
\|f\|_{s} \equiv\|f\|_{s-1}+\sum_{j=1}^{n}\left\|\frac{\partial f}{\partial x_{j}}\right\|_{s-1}<\infty .
$$

Note that the space $C^{k, Z}$ is locally equivalent to $\Lambda_{k+1}$ in the sense that any function in one of these spaces agrees on any bounded neighborhood with a function in the other. Similarly for the spaces $C^{k, \alpha}$ and $\Lambda_{k+\alpha}$. Since the Morse-Sard theorem is a local theorem, we are free to use either class. (The Lipschitz classes are more convenient for Theorem 2 since they free us from having to state the integer and noninteger cases separately.)

For any positive integer $k$, and $0<\alpha<\beta<1$, the following inclusions are well-known:

$$
\mathbf{C}^{k+1} \varsubsetneqq \mathbf{C}^{k, 1} \varsubsetneqq C^{k, Z} \varsubsetneqq \bigcap_{\gamma<1} C^{k, \gamma} \varsubsetneqq C^{k, \beta} \varsubsetneqq C^{k, \alpha} \varsubsetneqq C^{k} .
$$

In terms of the Lipschitz classes, if $0<s<t$, then

$$
\Lambda_{t} \varsubsetneqq \Lambda_{s} .
$$

The object of this paper is to prove 
Theorem 1 (Zygmund Morse-Sard Theorem). Let $n, m$ be positive integers with $n>m$. If $f \in C^{n-m, Z}\left(\mathbf{R}^{n}, \mathbf{R}^{m}\right)$ then the set of critical values of $f$ has Lebesgue measure zero.

This implies both the classical and Lipschitz Morse-Sard theorems.

In 1953, Dubovickii [3] proved that if $f: \mathbf{R}^{n} \rightarrow \mathbf{R}^{m}$ is $(n-m+1)$-times differentiable, then the set of its critical values has measure zero. Since differentiable functions need not be Zygmund, this is neither weaker nor stronger than theorem 1. However we note that a Zygmund function can be nowhere differentiable - e.g. the Weierstrass function $f(x)=\sum 2^{-k} \sin \left(2^{k} x\right)$.

Definition. A subset $E$ of $\mathbf{R}^{n}$ is a set of rank $r$ for $f$ if $\operatorname{rank} D f(x) \leq r$ for all $x \in E$.

Theorem 1 is an immediate consequence of

Theorem 2. Let $n, m$, and $r$ be nonnegative integers and suppose $n>m>r$. Let $E$ be a set of rank $r$ for $f: \mathbf{R}^{n} \rightarrow \mathbf{R}^{m}$, and set $s=(n-r) /(m-r)$.

If $f \in \Lambda_{s}$, then $f(E)$ has measure zero.

Remarks.

1. Theorem 1 follows from Theorem 2 by setting $r=m-1$.

2. Bates [1] proved theorem 2 in the case when $s$ is not an integer, making use of a slightly weaker version of the noninteger case in [7].

3. The simple form of theorem 2 is further evidence that the Lipschitz spaces $\Lambda_{s}$ are more natural than the smoothness classes $C^{s}$ for geometric analysis.

The idea of approach to Theorem 2 in the case when $s$ is an integer will be to follow the path of Morse and Sard within the Zygmund class. The key geometric lemma, stated below, permits us to obtain the same estimates as in the Lipschitz case. Then applying an idea of Bates to promote a "big oh" estimate to a "little oh" estimate will complete the proof.

Geometric Lemma. If $f: \mathbf{R}^{n} \rightarrow \mathbf{R}^{m}$ is Zygmund with Zygmund constant $C>0$, and $f(a)=f(b)=0$, then for all points $x$ belonging to the line segment joining a to $b$,

$$
|f(x)| \leq(C / 2)|b-a| .
$$

In Section 1 we present basic facts about the class $C^{k, Z}$ needed in the sequel (relegating the proof of a sharp Composition Theorem to Section 5). Section 2 contains the fundamental Morse Criticality Theorem for the spaces $\Lambda_{s}$. This is used in Section 3 to prove Theorem 2 in the special case of rank $r=0$. Then in Section 4 we use induction and Fubini's Theorem to arrive at the general case.

Acknowledgement. The author thanks S. Bates, P. Jones, S. Krantz, and D. Sullivan for helpful comments.

\section{BASIC PROPERTIES OF THE Zygmund Class}

This section contains proofs of basic facts about the class $C^{k, Z}$ that we will need in proving a Zygmund version of the Vanishing Lemma (see Section 2).

We will frequently use the fact that every Zygmund function $f$ has at least a $|t| \log (1 /|t|)$ modulus of continuity (e.g. [5]): for every compact set $K$, there is a constant $C>0$ such that for all $x \in K,|f(x+t)-f(x)| \leq C|t| \log (1 /|t|)$ for all $t$ such that $0<|t|<1$. Consequently a Zygmund function is always $\alpha$-Hölder for all $\alpha<1$. 
Product Lemma. If $f, g: \mathbf{R}^{n} \rightarrow \mathbf{R}$ are of class $C^{k, Z}, k \geq 0$, then so is the product $f g$.

Proof. First let $k=0$. For $t \in \mathbf{R}^{n}$ and $i=1,2$, define the first and second difference operators

$$
\Delta_{t}^{i}: C^{0}\left(\mathbf{R}^{n}, \mathbf{R}\right) \rightarrow C^{0}\left(\mathbf{R}^{n}, \mathbf{R}\right)
$$

by $\Delta_{t}^{1} f(x)=f(x+t)-f(x-t)$, and $\Delta_{t}^{2} f(x) \equiv \Delta_{t}^{1} \Delta_{t}^{1} f(x)=f(x+2 t)+f(x-$ $2 t)-2 f(x)$. Note that $f$ is Zygmund if and only if $\Delta_{t}^{2} f=O(|t|)$ as $|t| \rightarrow 0$.

Fix $t$ and let $T_{ \pm}(x)=x \pm t$.

One can check that

$$
\Delta_{t}^{1}(f g)=\left(\Delta_{t}^{1} f\right)\left(g \circ T_{-}\right)+\left(\Delta_{t}^{1} g\right)\left(f \circ T_{+}\right)
$$

and

$$
\Delta_{t}^{2}(f g)=f\left(\Delta_{t}^{2} g\right)+g\left(\Delta_{t}^{2} f\right)+\left[\left(\Delta_{t}^{1} f\right)\left(\Delta_{t}^{1} g\right)\right] \circ T_{+}+\left[\left(\Delta_{t}^{1} f\right)\left(\Delta_{t}^{1} g\right)\right] \circ T_{-} .
$$

Now if $f$ and $g$ are Zygmund, the first two terms are $O(|t|)$, and the third term is also $O(|t|)$ because it is a product of two factors, each of which is $O\left(|t|^{\alpha}\right)$ for $\alpha>1 / 2$.

This completes the proof for $k=0$. The statement follows for $k>0$ by induction from the product rule for differentiation.

The classes $C^{0, Z}$ and $\Lambda_{1}$ are unfortunately not closed under composition. In fact, even the composition of a Zygmund function with a $C^{1}$ function need not be Zygmund (see Section 5). However, if $C^{1}$ is strengthened to $C^{1, \alpha}$, we obtain the following statement, sufficient for the purposes of this paper:

Composition Lemma. For every $m, n, p \in Z^{+}$and $\alpha \in(0,1)$,

(a) $C^{0, Z}\left(\mathbf{R}^{n}, \mathbf{R}^{m}\right) \circ C^{1, \alpha}\left(\mathbf{R}^{p}, \mathbf{R}^{n}\right) \subset C^{0, Z}\left(\mathbf{R}^{p}, \mathbf{R}^{m}\right)$, and

(b) for every $k \in Z^{+}, C^{k, Z}\left(\mathbf{R}^{n}, \mathbf{R}^{m}\right) \circ C^{k, Z}\left(\mathbf{R}^{p}, \mathbf{R}^{n}\right) \subset C^{k, Z}\left(\mathbf{R}^{p}, \mathbf{R}^{m}\right)$.

Proof.

(a) This is an immediate corollary of the sharper Composition Theorem of Section 5 .

(b) This follows by induction using the chain rule, part (a), and the Product Lemma above.

Dennis Sullivan pointed out to the author that if $f$ is Zygmund and $g$ is $C^{1}$, then $f \circ g$ is Zygmund provided only that $D g$ satisfies a $1 / \log (1 / t)$ modulus of continuity, and this is sharp in the sense that no weaker modulus of continuity suffices. Discussion of these technicalities and the statement and proof of a sharp Composition Theorem are relegated to Section 5 .

Zygmund Inverse Function Theorem. If $f \in C^{k, Z}\left(\mathbf{R}^{n}, \mathbf{R}^{n}\right), k \geq 1, x \in \mathbf{R}^{n}$, and $D f_{x}$ is a linear isomorphism, then $f$ is invertible in a neighborhood of $x$ and $f^{-1}$ is of class $C^{k, Z}$.

Proof. The standard proof shows that $f^{-1}$ exists near $f(x)$, is $C^{k}$, and satisfies

$$
D\left(f^{-1}\right)=\operatorname{Inv} \circ D f \circ f^{-1}
$$


where Inv denotes the inverse operator on $\operatorname{GL}(n)$. In fact, for any $\alpha \in(0,1), f^{-1}$ is $C^{k+\alpha}$ by the $C^{k+\alpha}$ Inverse Function Theorem [7]. Therefore, since Inv is $C^{\infty}$, $D f$ is $C^{k-1, Z}$, and $f^{-1}$ is $C^{k+\alpha}$, the Composition Lemma implies that $D\left(f^{-1}\right)$ is $C^{k-1, Z}$.

Combining this with the $C^{k+\alpha}$ Inverse Function Theorem [7], we obtain the general

$\Lambda_{s}$ Inverse Function Theorem. If $f \in \Lambda_{s}\left(\mathbf{R}^{n}, \mathbf{R}^{n}\right), s>1, x \in \mathbf{R}^{n}$, and $D f_{x}$ is a linear isomorphism, then $f$ is invertible in a neighborhood of $x$ and $f^{-1}$ is of class $\Lambda_{s}$.

Many standard arguments now go through easily in the Zygmund class, e.g. the

Zygmund Preimage Theorem. If $k \geq 1, f \in C^{k, Z}\left(\mathbf{R}^{n}, \mathbf{R}\right), x \in \mathbf{R}^{n}, f(x)=0$, and $D f(x) \neq 0$, then there is a neighborhood $N$ of $x$ in $\mathbf{R}^{n}$ and $a C^{k, Z}(n-1)$ submanifold $S \subset \mathbf{R}^{n}$ such that

$$
f^{-1}(0) \cap N \subset S
$$

Proof. Use the standard argument (e.g. [4]) with the Zygmund Inverse Function Theorem.

To finish this section, we restate and prove the

Geometric Lemma. If $f: \mathbf{R}^{n} \rightarrow \mathbf{R}^{m}$ is Zygmund with Zygmund constant $C>0$, $a, b \in \mathbf{R}^{n}$, and $f(a)=f(b)=0$, then for all points $x$ belonging to the line segment $[a, b]$ joining a to $b$,

$$
|f(x)| \leq(C / 2)|b-a| .
$$

Proof. We are supposing that for all $x, h \in \mathbf{R}^{n}$,

$$
|f(x+h)+f(x-h)-2 f(x)| \leq C|h| .
$$

If $I$ is a line segment with endpoints $y$ and $z$ and midpoint $x$, this implies that

$$
|f(x)| \leq C|I| / 4+(|f(y)|+|f(z)|) / 2 .
$$

Let $L=|b-a|$. For $k=0,1,2, \ldots$, consider the collection $\mathcal{C}_{k}$ of closed dyadic subintervals of $[a, b]$, i.e. intervals with endpoints $a+\left(p / 2^{k}\right)(b-a)$ and $a+((p+$ 1) $\left./ 2^{k}\right)(b-a)$, where $p$ is an integer between 0 and $2^{k}-1$.

Define $B_{0}=\{a, b\}$, and for $k=1,2,3, \ldots$, define $B_{k}$ to be the finite set of points $x$ in $[a, b]$ such that $x$ is an endpoint of an interval of $\mathcal{C}_{k}$ but is an endpoint of no interval of $\mathcal{C}_{j}$ for any $j<k$. ( $B_{k}$ is the set of endpoints that "arise at stage $k$ ".)

Claim: For all $x \in B_{k},|f(x)| \leq C L\left(1-2^{-k}\right) / 2$.

We prove the claim below, but first note that this implies $|f(x)| \leq C L / 2$ for all $x$ in the dense set $\cup_{k} B_{k}$. Since $f$ is continuous, this yields the conclusion of the Geometric Lemma.

The claim is proved by simple induction. The case $k=0$ is simply our hypothesis that $f(a)=f(b)=0$. Let $k>0$ and suppose that for $j=0,1,2, \ldots, k-1$,

$$
|f(y)| \leq C L\left(1-2^{-j}\right) / 2 \text { for all } y \in B_{j} .
$$


Take $x \in B_{k}$. Then $x$ is the midpoint of some dyadic interval of length $L 2^{-(k-1)}$, with endpoints, say, $x^{\prime}$ and $x^{\prime \prime}$. By (1) above,

$$
|f(x)| \leq C L / 2^{k+1}+\left(\left|f\left(x^{\prime}\right)\right|+\left|f\left(x^{\prime \prime}\right)\right|\right) / 2 .
$$

By induction, since $x^{\prime}$ and $x^{\prime \prime}$ must appear at some stage before the $k$ th, $\left|f\left(x^{\prime}\right)\right|$ and $\left|f\left(x^{\prime \prime}\right)\right|$ are no more than $C L\left(1-2^{-(k-1)}\right) / 2$. Therefore

$$
\begin{aligned}
|f(x)| & \leq C L / 2^{k+1}+C L\left(1-2^{-(k-1)}\right) / 2 \\
& =C L\left(1-2^{-k}\right) / 2 .
\end{aligned}
$$

\section{A Zygmund version of the Morse Criticality Lemma}

In this section we take the fundamental step in the proof of Theorem 2 by proving the

Morse Criticality Theorem. Let $s \geq 1$ be a real number, $n \in Z^{+}, A \subset \mathbf{R}^{n}$. Then there is a countable collection $\left\{A_{i}\right\}$ of bounded subsets of $A$ such that

(i) $A=\cup A_{i}$, and

(ii) for every $f \in C^{1} \cap \Lambda_{s}\left(\mathbf{R}^{n}, \mathbf{R}\right)$ critical on $A$, and every $i$, there is a constant $c_{i}>0$ such that

$$
\text { for every } x, y \in A_{i}, \quad|f(x)-f(y)| \leq c_{i}|x-y|^{s} \text {. }
$$

In case $s=1$ or $s \notin Z^{+}$, this theorem appears in [7]. The only remaining case is the

Zygmund Morse Criticality Lemma. Let $n, k$ be positive integers and $A$ a subset of $\mathbf{R}^{n}$. Then there is a countable collection $\left\{A_{i}\right\}$ of bounded subsets of $A$ such that

(i) $A=\cup A_{i}$, and

(ii) for every $f \in C^{k, Z}\left(\mathbf{R}^{n}, \mathbf{R}\right)$ critical on $A$ and every $i$, there is a constant $c_{i}>0$ such that

$$
\text { for every } x, y \in A_{i}, \quad|f(x)-f(y)| \leq c_{i}|x-y|^{k+1} \text {. }
$$

This will be a direct consequence of the following lemma.

Definitions. We will say that the pair $(B, \phi)$ is a $C^{1}$ parametrized disk in $\mathbf{R}^{n}$ if for some integer $p, 1 \leq p \leq n, \phi$ is a $C^{1}$ embedding of $\mathbf{R}^{p}$ into $\mathbf{R}^{n}$ and $\phi(\Delta)=B$, where $\Delta$ is the closed unit ball in $\mathbf{R}^{p}$.

Given such a parametrized disk $(B, \phi)$, and two points $x, y \in B$, the parametric segment in $B$ from $x$ to $y$ is defined to be the image under $\phi$ of the line segment joining $\phi^{-1}(x)$ to $\phi^{-1}(y)$. 
Zygmund Morse Vanishing Lemma. Let $n$ be a positive integer, $k$ a nonnegative integer, and $A$ a subset of $\mathbf{R}^{n}$. Then there exists a countable set $A_{0}, a$ countable collection $\left\{A_{i}\right\}_{i=1}^{\infty}$ of bounded subsets of $\mathbf{R}^{n}$, and a countable collection $\left\{\left(B_{i}, \phi_{i}\right)\right\}_{i=1}^{\infty}$ of $C^{1}$ parametrized disks in $\mathbf{R}^{n}$ such that

(i) $A=\cup_{i=0}^{\infty} A_{i}$,

(ii) for each $i \in Z^{+}, A_{i} \subset B_{i}$, and

(iii) For every $f \in C^{k, Z}\left(\mathbf{R}^{n}, \mathbf{R}\right)$ vanishing on $A$, and every $i \in Z^{+}$, there is a positive constant $c_{i}$ such that

$$
|f(z)| \leq c_{i}|x-z|^{k}|x-y|
$$

whenever $x, y \in A_{i}$ and $z$ lies on the parametric segment in $B_{i}$ from $x$ to $y$.

We will call such a collection $\left\{A_{i}\right\}$ for a set $A$ a Morse decomposition of $A$.

Proof.

The proof is by double induction on $n$ and $k$. Let $\langle n, k\rangle$ stand for the statement of the theorem for $\mathbf{R}^{n}$ and $C^{k, Z}$. We will prove (a) $\langle n, 0\rangle$ for all $n$, (b) $\langle 1, k\rangle$ for all $k$, and (c) $\langle n-1, k\rangle$ and $\langle n, k-1\rangle$ imply $\langle n, k\rangle$.

(a) Proof of $\langle n, 0\rangle$ for all $n$.

If $A \subset \mathbf{R}^{n}$, let $A_{0}=\emptyset, B_{i}$ be the closed ball in $\mathbf{R}^{n}$ with center 0 and radius $i$, $\phi_{i}$ be the linear expansion $x \mapsto i x$ on $\mathbf{R}^{n}$, and $A_{i}=A \cap B_{i}$.

Given $f \in C^{k, Z}\left(\mathbf{R}^{n}, \mathbf{R}\right)$ vanishing on $A$, for each $i$ take $c_{i}$ to be the Zygmund constant of $f$ for the compact set $B_{i}$. The conclusion follows immediately from the Geometric Lemma of Section 1.

(b) Proof of $\langle 1, k\rangle$ for all $k$.

Let $A$ be a subset of $\mathbf{R}$. Denote by $A^{*}$ the set of condensation points of $A$; that is, $A^{*}$ is the set of points $x$ in $A$ such that every neighborhood of $x$ meets $A$ in uncountably many points. It is easy to show that $A_{0} \equiv A \backslash A^{*}$ is countable.

Now for $i>0$ let $A_{i}=A^{*} \cap(-i, i), B_{i}=[-i, i]$, and $\phi_{i}$ be the linear expansion by factor $i$. Given $f \in C^{k, Z}(\mathbf{R}, \mathbf{R})$, let $c_{i}^{\prime}$ be the Zygmund constant of $D^{k} f$ for the compact set $B_{i}$.

Since every point of $A_{i}$ is a limit point of $A_{i}$, and $f \in C^{1}$ vanishes on $A_{i}, D f$ must vanish at every point of $A_{i}$. Similarly, $D^{2} f, D^{3} f, \ldots, D^{k} f$ all vanish on $A_{i}$. then

By the Geometric Lemma applied to $D^{k} f$ on $B_{i}$, if $x, y \in A_{i}$ and $x<t<y$,

$$
\left|D^{k} f(t)\right| \leq c_{i}^{\prime}|x-y| \text {. }
$$

Hence, for any $z$ between $x$ and $y$, by integrating $D f(t) k$ times from $x$ to $z$ we obtain,

$$
\begin{aligned}
|f(z)| & =\left|\int_{x}^{z} \int_{x}^{t_{k}} \cdots \int_{x}^{t_{2}} D^{k} f\left(t_{1}\right) d t_{1} d t_{2} \ldots d t_{k}\right| \\
& \leq \int_{x}^{z} \int_{x}^{t_{k}} \cdots \int_{x}^{t_{2}} c_{i}^{\prime}|x-y| d t_{1} d t_{2} \ldots d t_{k} \\
& =c_{i}|x-z|^{k}|x-y|
\end{aligned}
$$

where $c_{i}=c_{i}^{\prime} / k !$.

(c) Induction step: we assume $\langle n-1, k\rangle$ and $\langle n, k-1\rangle$, and prove $\langle n, k\rangle$. 
Define

$U=\left\{x \in A:\right.$ for every $g \in C^{k, Z}\left(\mathbf{R}^{n}, \mathbf{R}\right)$ vanishing on $\left.A, \quad D g(x)=0\right\}$, and $V=A \backslash U$.

Since the union of two Morse decompositions is again a Morse decomposition, it suffices to prove the result for $U$ and $V$ separately.

We first address $U$. By our $\langle n, k-1\rangle$ hypothesis, there are subsets $U_{0}, U_{1}, U_{2}, \ldots$ of $U$ and $C^{1}$ parametrized disks $\left(B_{i}, \phi_{i}\right)$ such that $U_{0}$ is countable, $U=\cup U_{i}$, and $U_{i} \subset B_{i}$ for $i \geq 1$.

Moreover for any $h \in C^{k-1, Z}\left(\mathbf{R}^{n}, \mathbf{R}\right)$ vanishing on $V$, there are constants $c_{i}>0$ such that

$$
|h(t)| \leq c_{i}|x-t|^{k-1}|x-y|
$$

whenever $x, y \in U_{i}$ and $t$ lies on the parametric segment $[x, y]_{B_{i}}$ in $B_{i}$ from $x$ to $y$.

Now suppose $f \in C^{k}\left(\mathbf{R}^{n}, \mathbf{R}\right)$ vanishes on $U$. By definition of $U, f$ must be critical on $U$, so $D_{j} f \equiv \partial f / \partial x_{j}$ vanishes on $U$ for $j=1, \ldots, n$. Since these functions lie in $C^{k-1}\left(\mathbf{R}^{n}, \mathbf{R}\right)$, by above we know there exist constants $c_{i, j}, i=1,2,3, \ldots ; j=$ $1,2, \ldots, n$, so that

$$
\left|D_{j} f(t)\right| \leq c_{i, j}|x-t|^{k-1}|x-y|
$$

hence

$$
\|D f(t)\| \leq\left(\max _{j} c_{i, j}\right)|x-t|^{k-1}|x-y|
$$

whenever $x, y \in U_{i}$ and $t$ lies on the parametric segment $[x, y]_{B_{i}}$.

Now we need the following lemma, whose proof is straightforward and omitted:

Lemma 1. If $\Delta$ denotes the closed unit ball in $\mathbf{R}^{p}$ and $\phi$ is a $C^{1}$ embedding of $\Delta$ into $\mathbf{R}^{n}$, then there is a constant $\kappa$, depending on $\phi$, such that

$$
(1 / \kappa)|a-b| \leq|\phi(a)-\phi(b)| \leq \kappa|a-b|
$$

for all $a, b \in \Delta$.

Applying Lemma 1 to $\phi_{i}$ and $B_{i}$, we obtain a constant $k_{i}$ satisfying the conclusion of Lemma 1 , so that for all $x, z \in B_{i}$ and $t \in[x, z]_{B_{i}}$, we have

$$
\begin{aligned}
|x-t| & \leq k_{i}\left|\phi_{i}^{-1}(x)-\phi_{i}^{-1}(t)\right| \\
& \leq k_{i}\left|\phi_{i}^{-1}(x)-\phi_{i}^{-1}(z)\right| \\
& \leq k_{i}^{2}|x-z| .
\end{aligned}
$$

Therefore, letting $c_{i}^{\prime}=\left(\max _{j} c_{i, j}\right) k_{i}^{2(k-1)}$, and fixing $z$ on $[x, y]_{B_{i}},(2)$ yields

$$
|| D f(t) \| \leq c_{i}^{\prime}|x-z|^{k-1}|x-y|
$$

for all $t \in[x, z]_{B_{i}}$.

Now integrating $D f$ along $[x, y]_{B_{i}}$ from $x$ to $z$, we obtain

$$
|f(z)| \leq c_{i}^{\prime}|x-z|^{k-1}|x-y| L(x, z),
$$


where $L(x, z)$ denotes the length of the parametric segment $[x, z]_{B_{i}}$.

It follows from Lemma 1 that there is a constant $b_{i}>0$ such that $L(x, z) \leq$ $b_{i}|x-z|$ for all $x, z \in B_{i}$. Therefore we may let $c_{i}=c_{i}^{\prime} d_{i}$ and deduce that

$$
|f(z)| \leq c_{i}|x-z|^{k}|x-y|
$$

whenever $x, y \in U_{i}$ and $z$ lies on the parametric segment from $x$ to $y$ in $B_{i}$. This is our desired conclusion for $U$.

Turning now to $V$, note first that it suffices to show that $V$ satisfies the conclusion locally, i.e. in a neigborhood of each of its points. This is because a countable union of Morse decompositions is again a Morse decomposition.

So given $x \in V$, we show that, for some neighborhood $N$ of $x$ in $\mathbf{R}^{n}, V \cap N$ has an appropriate Morse decomposition.

Since $x \in V$, some $g \in C^{k, Z}\left(\mathbf{R}^{n}, \mathbf{R}\right)$ vanishing on $A$ is not critical at $x$. By the Zygmund Preimage Theorem, there exists a neighborhood $N$ of $x$ such that $A \cap N$, and hence $V \cap N$, is contained in a $C^{k, Z}(n-1)$-submanifold $S$ (namely, $S$ is the local zero set for $g$ ).

We can choose $S$ and an embedding $\psi \in C^{k, Z}\left(\mathbf{R}^{n-1}, \mathbf{R}^{n}\right)$ so that

$$
V \cap N \subset S=\psi(\Delta)
$$

where $\Delta$ denotes the closed unit ball in $\mathbf{R}^{n-1}$.

Writing $W=\psi^{-1}(V \cap N)$, we now have, by our $\langle n-1, k\rangle$ hypothesis:

(i) a collection $\left\{W_{i}\right\}_{i=0}^{\infty}$ of subsets of $W$, with $W_{0}$ countable and $W=\cup W_{i}$,

(ii) a collection $\left\{\left(D_{i}, \eta_{i}\right)\right\}$ of $C^{1}$ parametrized disks in $\mathbf{R}^{n-1}$ so that $W_{i} \subset D_{i}$ for all $i \geq 1$, and

(iii) for every $h \in C^{k, Z}\left(\mathbf{R}^{n-1}, \mathbf{R}\right)$ vanishing on $W$, and every $i \in Z^{+}$, a positive constant $d_{i}$ such that

$$
|h(z)| \leq d_{i}|x-z|^{k}|x-y|
$$

whenever $x, y \in W_{i}$ and $z$ lies on the parametric segment $[x, y]_{D_{i}}$ in $D_{i}$ joining $x$ to $y$.

Now define, for all $i$,

$$
V_{i}=\psi\left(W_{i}\right), B_{i}=\psi\left(D_{i}\right), \text { and } \phi_{i}=\psi \circ \eta_{i}
$$

Then the following facts are immediate:

(i') $V_{0}$ is countable and $\cup V_{i}=V \cap N$, and

(ii') each pair $\left(B_{i}, \phi_{i}\right)$ is a $C^{1}$ parametrized disk in $\mathbf{R}^{n}$ with $V_{i} \subset B_{i}$ for all $i \geq 1$.

Moreover,

(iii') if $f \in C^{k, Z}\left(\mathbf{R}^{n}, \mathbf{R}\right)$ vanishes on $V \cap N$, then $f \circ \psi \in C^{k, Z}\left(\mathbf{R}^{n-1}, \mathbf{R}\right)$ vanishes on $W$.

If we pick an arbitrary $x^{\prime}, y^{\prime} \in V_{i}$ and $z^{\prime}$ on the parametric segment $\left[x^{\prime}, y^{\prime}\right]_{B_{i}}$, then there are points $x, y, z$ in $D_{i}$ such that $\psi(x)=x^{\prime}, \psi(y)=y^{\prime}, \psi(z)=z^{\prime}$, $x, y \in W_{i}$, and $z \in[x, y]_{D_{i}}$.

Hence, by (iii) above, there exist constants $d_{i}$, depending on $f$ but independent of $x, y, z$, such that

$$
|f \circ \psi(z)| \leq d_{i}|x-z|^{k}|x-y|
$$


Applying Lemma 1 to the embedding $\psi$, we obtain a constant $k_{i}>0$ such that

$$
|a-b| \leq k_{i}|\psi(a)-\psi(b)|
$$

for all $a, b \in \Delta$. In particular, $|x-z| \leq k_{i}\left|x^{\prime}-z^{\prime}\right|$ and $|x-y| \leq k_{i}\left|x^{\prime}-y^{\prime}\right|$, so that

$$
\left|f\left(z^{\prime}\right)\right| \leq d_{i} k_{i}^{k+1}\left|x^{\prime}-z^{\prime}\right|^{k}\left|x^{\prime}-y^{\prime}\right| .
$$

This completes the inductive step, and the proof.

Proof of Zygmund Morse Criticality Lemma.

Let $\left\{A_{i}^{\prime}\right\}$ be the Morse decomposition given by the Morse Vanishing Lemma for the pair $\langle n, k-1\rangle$. Let $\left\{\left(B_{i}, \phi_{i}\right)\right\}$ be the corresponding $C^{1}$ parametrized disks. We check (ii).

Given $f \in C^{k, Z}\left(\mathbf{R}^{n}, \mathbf{R}\right)$ critical on $A$, this means that for each $j=1, \ldots, n, D_{j} f$ lies in $C^{k-1, Z}$ and vanishes on $A$.

The Morse Vanishing Lemma now guarantees us constants $c_{i, j}>0$ such that

$$
\left|D_{j} f(z)\right| \leq c_{i, j}|x-z|^{k-1}|x-y|
$$

whenever $x, y \in A_{i}^{\prime}$ and $z \in[x, y]_{B_{i}}$.

By Lemma 1 , as in the preceding argument, there is a constant $k_{i}>0$ such that $|x-z| \leq k_{i}^{2}|x-y|$ so long as $x, y \in A_{i}^{\prime}$ and $z \in[x, y]_{B_{i}}$. Therefore $|D f(z)|$ is controlled by a constant times $|x-y|^{k}$. Integrating $D f$ along $[x, y]_{B_{i}}$ from $x$ to $z$ gives, as in the proof of the Vanishing Lemma, a constant $c_{i}>0$, depending only on $f$ and $i$, such that

$$
|f(x)-f(y)| \leq c_{i}|x-y|^{k+1}
$$

whenever $x, y \in A_{i}^{\prime}$.

We now have a collection $\left\{A_{i}^{\prime}\right\}_{i=1}^{\infty}$ satisfying the desired conclusions, except that the union $\cup A_{i}^{\prime}$ omits the countable subset $A_{0}^{\prime}$ of $A$. Let $\left\{A_{i}\right\}=\left\{A_{i}^{\prime}\right\} \cup\{\{x\}$ : $\left.x \in A_{0}^{\prime}\right\}$. This countable collection now suffices, since when $A_{i}$ is a singleton, the condition (ii) is trivially true.

\section{The Rank Zero Lemmas}

The following lemmas will establish the rank zero case of Theorem 2. In section 4 we repeat the argument in [7] and [10] deducing the general case from this one.

First Rank Zero Lemma. Let $n>m, f \in \Lambda_{n / m}\left(\mathbf{R}^{n}, \mathbf{R}^{m}\right)$, and $A \subset \mathbf{R}^{n}$ be a set of rank zero for $f$. If $A$ has measure zero, then $f(A)$ has measure zero.

Proof. By the Morse Criticality Theorem, there is a Morse decomposition $A=\cup A_{i}$ such that every component of $f$, and therefore $f$ itself, satisfies

$$
|f(x)-f(y)| \leq c_{i}|x-y|^{n / m}
$$

for some $c_{i}>0$ and every $x, y \in A_{i}, i>0$.

Fix $j$ and write $B=A_{j}$ and $c=c_{j}$; we show that $f(B)$ has measure zero. Let $\epsilon>0$ be given. Since $B$ has measure zero, it is contained in the union $\cup B_{i}$ of balls in $\mathbf{R}^{n}$ chosen such that $\sum\left|B_{i}\right|^{n}<\epsilon / c^{m}$, where $\left|B_{i}\right|$ denotes the diameter of $B_{i}$. 
By (2), for each $i$,

$$
\left|f\left(B \cap B_{i}\right)\right| \leq c\left|B \cap B_{i}\right|^{n / m} .
$$

Letting $l_{m}$ denote $m$-dimensional Lebesgue measure, we therefore have

$$
\begin{aligned}
l_{m}(f(B)) & \leq \sum l_{m}\left(f\left(B \cap B_{i}\right)\right) \leq \sum\left|f\left(B \cap B_{i}\right)\right|^{m} \\
& \leq \sum\left(c\left|B \cap B_{i}\right|^{n / m}\right)^{m} \\
& \leq c^{m} \sum\left|B_{i}\right|^{n}<\epsilon .
\end{aligned}
$$

Hence $l_{m}(f(B))=0$.

In the next lemma we invoke an argument of Bates [1] to remove the requirement in the First Rank Zero Lemma that $A$ have measure zero.

Second Rank Zero Lemma. Let $n>m, f \in \Lambda_{n / m}\left(\mathbf{R}^{n}, \mathbf{R}^{m}\right)$, and $A \subset \mathbf{R}^{n}$ be a set of rank zero for $f$.

Then $f(A)$ has measure zero.

Proof. Let $\left\{A_{i}\right\}$ be a Morse decomposition for $A$ as provided by the Morse Criticality Lemma. (Without loss of generality, we assume that $l_{n}\left(A_{i}\right)<\infty$ for each $i$.) Fix $j \geq 1$ and write $B=A_{j}$; our job is to show that $l_{m}(f(B))=0$.

By the Morse Criticality Lemma applied to each component of $f$, there is a constant $c>0$ such that

$$
|f(x)-f(y)| \leq c|x-y|^{n / m}
$$

for all $x, y \in B$.

We may assume that $B$ is measurable, since replacing $B$ by its closure preserves condition (3). Hence, by the Lebesgue Density Theorem, almost every point of $B$ is a density point. That is, we can write $B=D \cup E$, where $l_{n}(E)=0$ and every point of $D$ is a density point of $D$. The First Rank Zero Lemma shows that $l_{m}(f(E))=0$; it remains to show that $l_{m}(f(D))=0$.

The following lemma is convenient:

Lemma 2. If $n \geq 1, P \geq 2$ are integers, $D \subset \mathbf{R}^{n}$ is measurable, and $Q$ is a cube in $\mathbf{R}^{n}$ satisfying

$$
\frac{l_{n}(D \cap Q)}{l_{n}(Q)} \geq 1-P^{-n}
$$

then for every $x, y \in D \cap Q$, there is a sequence $x_{0}, \ldots, x_{P}$ of points in $D \cap Q$ such that $x_{0}=x, x_{P}=y$, and

$$
\left|x_{i}-x_{i+1}\right|<2|Q| / P
$$

for $i=0, \ldots, P-1$.

Lemma 2 is proved below, but first we finish the proof of the Second Rank Zero Lemma. First choose an arbitrary $P \geq 2$. Since every point of $D$ is a density point, there is a positive function $\delta: D \rightarrow \mathbf{R}$ so that any cube $Q(x, \delta)$ with center $x$ and side length $\delta<\delta(x)$ satisfies (4). 
Let $Q$ be any such cube. Then for every $x, y \in D \cap Q$,

$$
\begin{aligned}
|f(x)-f(y)| & \leq\left|f\left(x_{0}\right)-f\left(x_{1}\right)\right|+\cdots+\left|f\left(x_{P-1}\right)-f\left(x_{P}\right)\right| \\
& \leq c\left|x_{0}-x_{1}\right|^{n / m}+\cdots+c\left|x_{P-1}-x_{P}\right|^{n / m}(\text { by }(3)) \\
& \leq c P(2|Q| / P)^{n / m} \\
& =c 2^{n / m} P^{1-(n / m)}|Q|^{n / m} .
\end{aligned}
$$

This means that $|f(D \cap Q)| \leq c 2^{n / m} P^{1-(n / m)}|Q|^{n / m}$, so

$$
\begin{aligned}
l_{m}(f(D \cap Q)) & \leq|f(D \cap Q)|^{m} \leq c^{m} 2^{n} P^{m-n}|Q|^{n} \\
& \leq c^{m} 2^{2 n} P^{m-n} l_{n}(Q) .
\end{aligned}
$$

Since $\{Q(x, \delta): x \in D, \delta<\delta(x)\}$ is a Vitali family for $D$, there is a countable subcollection $\left\{Q_{i}\right\}$ such that $l_{n}\left(D \backslash \cup Q_{i}\right)=0$ and $\sum l_{n}\left(Q_{i}\right)<2 l_{n}(D) .\left(l_{n}(D)<\infty\right.$ since $D$ is bounded.) By the First Rank Zero Lemma, $l_{m}\left(f\left(D \backslash \cup Q_{i}\right)\right)=0$.

Hence

$$
\begin{aligned}
l_{m}(f(D)) & \leq \sum l_{m}\left(f\left(Q_{i}\right)\right) \\
& \leq \sum c^{m} 2^{2 n} P^{m-n} l_{n}\left(Q_{i}\right) \\
& <2^{2 n+1} c^{m} P^{m-n} l_{n}(D) .
\end{aligned}
$$

Since $P$ is arbitrary, $l_{m}(f(D))$ must vanish, and this completes the proof.

Proof of Lemma 2. Assume (4) and $x, y \in D \cap Q$. Clearly the line segment $L$ joining $x$ to $y$ can be covered by at most $P$ subcubes of $Q$ of diameter $|Q| / P$. By (4), each of these subcubes must contain a point of $D \cap Q$, so choosing a point in each yields the desired sequence.

\section{Proof of Theorem 2}

For nonnegative integers $n>m>r$, let $s=(n-r) /(m-r)$. We assume $f \in \Lambda_{s}\left(\mathbf{R}^{n}, \mathbf{R}^{m}\right)$ and $E \subset \mathbf{R}^{n}$ is a set of rank $r$ for $f$.

For $i=0,1,2, \ldots, r$, define $R_{i}=\{x \in E: \operatorname{rank} D f(x)=i\}$. We need to show that $l_{m}\left(f\left(R_{i}\right)\right)=0$ for each $i$.

The case $i=0$ is covered by the Second Rank Zero Theorem of the previous section, so we now fix $i \geq 1$. It will suffice to find, for every $p \in R_{i}$, a neighborhood $U$ of $p$ such that $l_{m}\left(f\left(\bar{U} \cap R_{i}\right)\right)=0$.

By means of a standard argument (e.g. as in [4]) using the $\Lambda_{s}$ Inverse Function Theorem, we can find coordinates in some neighborhood $U$ of $p$ so that

$$
f\left(x_{1}, \ldots, x_{n}\right)=\left(x_{1}, \ldots, x_{i}, g\left(x_{1}, \ldots, x_{n}\right)\right),
$$

where $g \in \Lambda_{s}\left(\mathbf{R}^{n}, \mathbf{R}^{m-i}\right)$.

In these coordinates,

$$
D f(x)=\left(\begin{array}{cc}
I d_{i} & 0 \\
* & D\left(g\left[x_{1}, \ldots, x_{i}\right]\right)
\end{array}\right),
$$


where $x=\left(x_{1}, \ldots, x_{n}\right), I d_{i}$ is the $i \times i$ identity matrix, and $g\left[x_{1}, \ldots, x_{i}\right]$ denotes the function $\mathbf{R}^{n-i} \rightarrow \mathbf{R}^{m-i}$ defined by

$$
g\left[x_{1}, \ldots, x_{i}\right]:\left(x_{i+1}, \ldots, x_{n}\right) \mapsto g\left(x_{1}, \ldots, x_{n}\right)
$$

By definition of $R_{i}$, if $x \in U \cap R_{i}$, then $\operatorname{rank} D\left(g\left[x_{1}, \ldots, x_{i}\right]\right)=0$.

Define the "cross-section" of a set $A \subset \mathbf{R}^{n}$ at $\left(x_{1}, \ldots, x_{i}\right) \in \mathbf{R}^{i}$ by

$$
A\left[x_{1}, \ldots, x_{i}\right]=\left\{\left(x_{i+1}, \ldots, x_{n}\right) \in \mathbf{R}^{n-i}:\left(x_{1}, \ldots, x_{n}\right) \in A\right\}
$$

In these terms, it is easy to check that, for $x \in U \cap R_{i}, g\left[x_{1}, \ldots, x_{i}\right]$ maps the rank 0 set $\left(U \cap R_{i}\right)\left[x_{1}, \ldots, x_{i}\right] \subset \mathbf{R}^{m-i}$ onto the set $\left(f\left(U \cap R_{i}\right)\right)\left[x_{1}, \ldots, x_{i}\right] \subset \mathbf{R}^{m-i}$.

Now, because $(n-i) /(m-i) \leq(n-r) /(m-r)=s$, we have

$$
g\left[x_{1}, \ldots, x_{i}\right] \in \Lambda_{s}\left(\mathbf{R}^{n-i}, \mathbf{R}^{m-i}\right) \subset \Lambda_{(n-i) /(m-i)}\left(\mathbf{R}^{n-i}, \mathbf{R}^{m-i}\right) .
$$

Therefore, by the Second Rank Zero Lemma applied to $g\left[x_{1}, \ldots, x_{i}\right]$ on $(U \cap$ $\left.R_{i}\right)\left[x_{1}, \ldots, x_{i}\right]$, we obtain

$$
l_{m-i}\left(\left(f\left(U \cap R_{i}\right)\right)\left[x_{1}, \ldots, x_{i}\right]\right)=0 .
$$

Since this holds for every $x \in U \cap R_{i}$, we may apply Fubini's Theorem for $\mathbf{R}^{m}=$ $\mathbf{R}^{i} \times \mathbf{R}^{m-i}$ to deduce

$$
l_{m}\left(f\left(U \cap R_{i}\right)\right)=0 .
$$

\section{Moduli of Continuity and a Composition Theorem}

In this section we discuss, as promised in Section 1, the question of how smooth a function $g$ must be so that $f \circ g$ is guaranteed to be Zygmund if $f$ is Zygmund. For this we need to discuss moduli of continuity.

Definition. If $(X, d),\left(Y, d^{\prime}\right)$ are metric spaces and $f: X \rightarrow Y$ is continuous, the modulus of continuity $m_{f}$ of $f$ is defined to be the function

$$
m_{f}(t)=\sup \left\{d^{\prime}(f(x), f(y)): d(x, y) \leq t\right\}
$$

for $t \geq 0$. (This function might in general be infinite for some or all $t>0$.)

A function $\alpha:[0, \infty) \rightarrow[0, \infty]$ is called a modulus of continuity if $\alpha$ is monotone, continuous, and $\alpha(0)=0$.

These definitions are consistent because of the fact (whose proof we omit) that for every modulus of continuity $\alpha$, there is a (possibly infinite-dimensional) metric space $(X, d)$ and a continuous function $f: X \rightarrow \mathbf{R}$ such that $m_{f}=\alpha$.

We now restrict attention to functions defined on Euclidean spaces. Since we are interested in local smoothness properties, we are free to confine our attention to continuous functions $f: \mathbf{R}^{n} \rightarrow \mathbf{R}^{m}$ with compact support (i.e. locally constant outside a compact set). Such functions always have a finite modulus of continuity. 
Definition. A function $f$ is majorized by a modulus of continuity $\alpha$ if $m_{f} \leq \alpha$. Equivalently,

$$
|f(x)-f(y)| \leq \alpha(|x-y|)
$$

for all $x, y$.

Example. If $f$ is majorized by $\alpha(t)=c t$, then $f$ is Lipschitz; if by $\alpha(t)=c t^{s}$, then $f$ is $s$-Hölder.

Only certain functions $\alpha$ can arise as moduli of continuity for functions $f: \mathbf{R}^{n} \rightarrow$ $\mathbf{R}^{m}$ of compact support - these are the subadditive functions, i.e. those satisfying

$$
\alpha(s+t) \leq \alpha(s)+\alpha(t)
$$

for all $s, t \in[0, \infty)$.

It is easy to check that for any such $f, m_{f}$ is subadditive and has compact support; conversely, if $\alpha$ is a subadditive modulus of continuity with compact support, then $\alpha=m_{f}$ for some $f$-namely, $f(t)=\alpha(t)$ for $t \geq 0, f(t)=0$ otherwise.

Definition. For $k=0,1,2, \ldots$ and $\alpha$ a modulus of continuity, we say that

$$
f \in C^{k, \alpha}
$$

if $f \in C^{k}$ and $D^{k} f$ is locally majorized by a multiple of $\alpha$. If $f$ also has compact support, we write

$$
f \in C_{c}^{k, \alpha} \text {. }
$$

Suppose $\alpha$ and $\beta$ are moduli of continuity, and for some $C>1$ and all $t>0$,

$$
1 / C \leq \frac{\alpha(t)}{\beta(t)} \leq C
$$

We then think of $\alpha$ and $\beta$ as equivalent because $C^{k, \alpha}=C^{k, \beta}$. This defines an equivalence relation $\sim$ on the set of all moduli of continuity. There is a natural partial order $\preccurlyeq$ :

$$
\alpha \preccurlyeq \beta \text { if } \alpha \sim \beta \text { or } \alpha \prec \beta,
$$

where

$$
\alpha \prec \beta \text { means } \alpha(t) / \beta(t) \rightarrow \infty \text { as } t \rightarrow 0^{+} \text {. }
$$

Clearly, if $\alpha \preccurlyeq \beta$, then $C_{c}^{k, \beta} \subset C_{c}^{k, \alpha}$.

Now we can state the

Composition Theorem. Let

$$
\lambda(t)= \begin{cases}\frac{1}{\log (1 / t)} & \text { for } 0<t \leq e^{-2} \\ 1 / 2 & \text { for } t \geq e^{-2}\end{cases}
$$

and $\lambda(0)=0$

Then $\lambda$ is a subadditive modulus of continuity. Moreover,

(a) If $f \in C^{0, Z}\left(\mathbf{R}^{n}, \mathbf{R}^{m}\right)$ and $g \in C^{1, \lambda}\left(\mathbf{R}^{p}, \mathbf{R}^{n}\right)$, then $f \circ g \in C^{0, Z}$, and 
(b) There exists $f \in C^{Z}(\mathbf{R}, \mathbf{R})$ such that for every subadditive modulus of continuity $\alpha \prec \lambda$, there is a function $g \in C_{c}^{1, \alpha}(\mathbf{R}, \mathbf{R})$ such that $f \circ g \notin C^{Z}$.

Proof. One easily checks that $\lambda$ is continuous, monotone, and concave, hence subadditive.

Proof of (a). Given $f \in C^{Z}, g \in C^{1+\lambda}$, we show that the composition $f \circ g$ is Zygmund by a straightforward estimate of the second difference.

Fix a convex compact set $K$ in $\mathbf{R}^{p}$. Denote by $A$ the Zygmund constant of $f$ on $g(K)$, and choose $B$ so that $|D g(x)-D g(y)| \leq B \lambda(|x-y|)$ for $x, y \in K$. Recall that $f$ must satisfy a $t \log (1 / t)$ modulus of continuity. That is, if we let $\beta(t)=t \log (1 / t)$ for $0<t<1 / 2$, and $\beta(t)=(\log 2) / 2$ otherwise, then there exists $L>0$ such that $|f(x)-f(y)| \leq L \beta(|x-y|)$ for all $x, y \in K$.

Applying Taylor's theorem to the components $g_{i}$ of $g$, we find that

$$
g_{i}(x+t)=g_{i}(x)+D g_{i}\left(\xi_{i}\right) t,
$$

where $\xi_{i}$ is some point on the line segment $[x, x+t], x, x+t \in K$.

Hence

$$
\begin{aligned}
|g(x+t)-g(x)-D g(x) t| & \leq \sum_{i}\left|g_{i}(x+t)-g_{i}(x)-D g_{i}(x) t\right| \\
& \leq \sum\left|D g_{i}\left(\xi_{i}\right) t-D g_{i}(x) t\right| \\
& \leq n B \lambda(|t|)|t| .
\end{aligned}
$$

Now we estimate:

$$
\begin{aligned}
& |f \circ g(x+t)+f \circ g(x-t)-2 f \circ g(x)| \\
& \leq|f(g(x)+D g(x) t)+f(g(x)-D g(x) t)-2 f(g(x))| \\
& \quad \quad+|f(g(x)+D g(x) t)-f(g(x+t))|+|f(g(x)-D g(x) t)-f(g(x-t))| \\
& \leq A\|D g(x)\||t|+L \beta(|g(x+t)-g(x)-D g(x) t|) \\
& \quad+L \beta(|g(x-t)-g(x)-D g(x)(-t)|) \\
& \leq A\|D g(x)\||t|+2 L \beta(n B \lambda(|t|)|t|) .
\end{aligned}
$$

The first term on the right is $O(|t|)$ as $|t| \rightarrow 0$ since $\|D g\|$ is bounded on $K$. That the second term is also $O(|t|)$, with constants depending only on $K, A, B$ and $L$, is easily verified using the definitions of $\beta$ and $\lambda$, and the fact that $(\log (C /(-\log |t|))) / \log |t|$ tends to zero as $|t| \rightarrow 0$.

Proof of (b). Let $f(x)=\sum_{k=0}^{\infty} 2^{-k} \sin \left(2^{k} x\right)$.

It is known that $f$ is Zygmund (e.g. [5]), and we use the fact that there exists $C>0$ such that

$$
f(x) \geq C x \log (1 / x)
$$

for $0<x<e^{-2}$.

Given the subadditive modulus of continuity $\alpha$, extend $\alpha$ to all of $\mathbf{R}$ by letting it have value zero on $\mathbf{R}^{-}$. Construct $\hat{\alpha}$ by reflection in the line $x=1$ :

$$
\hat{\alpha}(t)= \begin{cases}\alpha(t) & \text { for } t \leq 1 \\ \alpha(1-t) & \text { for } t>1\end{cases}
$$


Now define $g(x)=\int_{0}^{x} \hat{\alpha}(t) d t$. Evidently $g$ has compact support since $\hat{\alpha}$ is zero outside of $[0,2]$. To show that $g \in C^{1, \alpha}$, we need only show that $\hat{\alpha}$ is majorized by $\alpha$.

Case 1: $0 \leq x<y \leq 1$. Then

$$
|\hat{\alpha}(x)-\hat{\alpha}(y)|=|\alpha(x)-\alpha(y)|=|\alpha(x)-\alpha(x+(y-x))| \leq \alpha(y-x)
$$

by subadditivity.

Case 2: $1 \leq x<y$. Similar. Then

Case 3: $x<1<y$. Suppose $x$ is closer to 1 than $y$ (otherwise argue similarly).

$$
|\hat{\alpha}(x)-\hat{\alpha}(y)| \leq|\hat{\alpha}(1)-\hat{\alpha}(y)| \leq \alpha(|1-y|)
$$

as before, and by monotonicity this is at most $\alpha(|x-y|)$.

It now remains to establish that $f \circ g$ is not Zygmund. Choose a positive integer $N$. Since $\alpha \prec \lambda$, there is $\epsilon=\epsilon(N)<e^{-2}$ such that $0<x<\epsilon$ implies $\alpha(t) \geq N \lambda(t)$.

For such $x$,

$$
\begin{aligned}
|f(g(x))+f(g(-x))-2 f(g(0))| & =|f(g(x))| \\
& \geq C g(x) \log (1 / g(x)) \\
& \geq C\left(\int_{0}^{x} N \lambda(t) d t\right)\left(-\log \int_{0}^{x} N \lambda(t) d t\right)
\end{aligned}
$$

since the function $t \log (1 / t)$ is monotone on $\left(0, e^{-2}\right)$,

$$
=C N\left(\int_{0}^{x} \lambda(t) d t\right)\left[-\log N-\log \int_{0}^{x} \lambda(t) d t\right] .
$$

If we choose $\epsilon^{\prime} \leq \epsilon$ so small that $x<\epsilon^{\prime}$ implies

$$
\int_{0}^{x} \lambda(t) d t<1 / N^{2}
$$

then

$$
-\log \int_{0}^{x} \lambda(t) d t>2 \log N
$$

and so

$$
f(g(x)) \geq(C N / 2)\left(\int_{0}^{x} \lambda(t) d t\right)\left(-\log \int_{0}^{x} \lambda(t) d t\right) .
$$

Now denote by $\bar{\lambda}(x)$ the average value $(1 / x) \int_{0}^{x} \lambda(t) d t$ of $\lambda$ on the interval $[0, x]$.

We now obtain

$$
\begin{aligned}
f(g(x)) / x & \geq(C N / 2)\{\bar{\lambda}(x)[-\log \bar{\lambda}(x)+\log (1 / x)]\} \\
& =(C N / 2)\{\bar{\lambda}(x) \log (1 / \bar{\lambda}(x))+\bar{\lambda}(x) \log (1 / x)\}
\end{aligned}
$$

The first term on the right tends to zero as $x$ tends to zero. The second term is

$$
\frac{\int_{0}^{x} \lambda(t) d t}{x \lambda(x)}
$$


One easily checks, using L'Hopital's rule and the definition of $\lambda$, that this tends to one as $x$ tends to zero.

Hence, for $x$ sufficiently small,

$$
\Delta_{(x / 2)}^{2} f(0)=f(g(x)) \geq(C N / 4) x
$$

Since $N$ was chosen arbitrarily, this means that $f(g(x)) / x \rightarrow+\infty$ as $x \rightarrow 0^{+}$, so $f \circ g$ is not Zygmund.

\section{REFERENCES}

1. S. Bates, Toward a precise smoothness hypothesis in Sard's Theorem, Proc. Amer. Math. Soc. 117 (1993), 279-283.

2. __ On the image size of singular maps, II, Duke Math. J. 68 (1992), 463-476.

3. A. Ya. Dubovickii, On differentiable mappings of an $n$-dimensional cube into a $k$-dimensional cube, Mat. Sbornik (N.S.) 32 (1953), 443-464. (Russian)

4. V. Guillemin and A. Pollack, Differential Topology, Prentice-Hall, 1974.

5. S. Krantz, Lipschitz spaces, smoothness of functions, and approximation theory, Expositiones Mathematicae 3 (1983), 193-260.

6. A.P. Morse, The behavior of a function on its critical set, Ann. of Math. 40, 62-70.

7. A. Norton, A critical set with non-null image has large Hausdorff dimension, Trans. Amer. Math. Soc. 296 (1986), 367-376.

8. Functions not constant on fractal quasi-arcs of critical points, Proc. Amer. Math. Soc. 106 (1989), 397-406.

9. A. Norton and D. Sullivan, Wandering domains and invariant conformal structures for mappings of the 2-torus, preprint.

10. A. Sard, The measure of the critical values of differentiable maps, Bull. Amer. Math. Soc. 48 (1942), 883-890.

11. E. Stein, Singular Integrals and Differentiability Properties of Functions, Princeton Univ. Press, 1970.

12. D. Varberg, On differentiable transformations in $R^{n}$, Amer. Math. Monthly 73 (1966), 111114.

13. H. Whitney, A function not constant on a connected set of critical points, Duke Math. J. 1 (1935), 514-517. 